\title{
Modelling of Applied Magnetic Field and Thermal Radiations Due to the Stretching of Cylinder
}

\author{
Muhammad Tamoor ${ }^{1,2}{ }^{(D}$, Muhammad Kamran ${ }^{3}\left(\mathbb{D}\right.$, Sadique Rehman ${ }^{4}$, Aamir Farooq ${ }^{5, *}$, Rewayat Khan ${ }^{5}$, \\ Jung Rye Lee ${ }^{6}$ and Dong Yun Shin ${ }^{7, *}$
}

1 CAS Key Laboratory of Green Process and Engineering \& State Key Laboratory of Biochemical Engineering, Institute of Process Engineering, Chinese Academy of Sciences, Beijing 100190, China; quaid.taimoor@gmail.com

2 College of Chemical Engineering, University of Chinese Academy of Sciences, 19 A Yuquan Road, Beijing 100049, China

3 Department of Mathematics, COMSATS University Islamabad, Wah Campus 47040, Pakistan; getkamran@gmail.com

4 Department of Mathematics, Islamia College Peshawar, Peshawar 25000, Pakistan; sadiquerehmanmaths@gmail.com

5 Department of Mathematics, Abbottabad University of Science and Technology, Abbottabad 22010, Pakistan; rewayat.khah@gmail.com

6 Department of Mathematics, Daejin University, Kyunggi 11159, Korea; jrlee@daejin.ac.kr

7 Department of Mathematics, University of Seoul, Seoul 02504, Korea

* Correspondence: aamirf88@aust.edu.pk (A.F.); dyshin@uos.ac.kr (D.Y.S.)

Citation: Tamoor, M.; Kamran, M.; Rehman, S.; Farooq, A.; Khan, R.; Lee, J.R.; Shin, D.Y. Modelling of Applied Magnetic Field and Thermal Radiations Due to the Stretching of Cylinder. Processes 2021, 9, 1077. https://doi.org/10.3390/pr9061077

Academic Editors: Kosmas Ellinas and Panagiotis Dimitrakellis

Received: 24 May 2021

Accepted: 14 June 2021

Published: 21 June 2021

Publisher's Note: MDPI stays neutral with regard to jurisdictional claims in published maps and institutional affiliations.

Copyright: (c) 2021 by the authors. Licensee MDPI, Basel, Switzerland. This article is an open access article distributed under the terms and conditions of the Creative Commons Attribution (CC BY) license (https:// creativecommons.org/licenses/by/ $4.0 /)$.

\begin{abstract}
In this study, a numerical approach was adopted in order to explore the analysis of magneto fluid in the presence of thermal radiation combined with mixed convective and slip conditions. Using the similarity transformation, the axisymmetric three-dimensional boundary layer equations were reduced to a self-similar form. The shooting technique, combined with the Range-Kutta-Fehlberg method, was used to solve the resulting coupled nonlinear momentum and heat transfer equations numerically. When physically interpreting the data, some important observations were made. The novelty of the present study lies in finding help to control the rate of heat transfer and fluid velocity in any industrial manufacturing processes (such as the cooling of metallic plates). The numerical results revealed that the Nusselt number decrease for larger Prandtl number, curvature, and convective parameters. At the same time, the skin friction coefficient was enhanced with an increase in both slip velocity and convective parameter. The effect of emerging physical parameters on velocity and temperature profiles for a nonlinear stretching cylinder has been thoroughly studied and analyzed using plotted graphs and tables.
\end{abstract}

Keywords: magnetic field; thermal radiations; mixed convection; nonlinear stretching; velocity slip; thermal slip

\section{Introduction}

Computational experiments in natural convection heat and mass transfer through thermal radiative and hydromagnetic flow have recently overwhelmed numerous scientists due to their importance in industrial technology [1]. This research has various industrial applications including the aerodynamic extrusion of plastic sheets, the cooling of metallic plates, energy storage devices, oil reservoirs, and micro magnetohydrodynamics (MHD) pumps [2].

To begin, [3] investigated the boundary layer flow (BLF) on continuous solid surfaces. They investigated whether the new boundary layer class had a different solution sustainability than the BLF on finite surfaces. In the presence of physical conditions, such as the stretching of surfaces, significant properties of flow and heat transfer have been investigated in the literature. A hybrid analytical and experimental investigation related to 
velocity-temperature fields in the boundary layer due to the stretching of surfaces has been conducted by [4]. Ref. [5] later applied this definition to the BLF in 1970. Crane studied fluid flow over the stretchable surface and found a similar solution for two-dimensional steady flow. Additionally, [6] examined the two-dimensional model and proposed similar solutions for the stretching of both the channel and cylinder. Ref. [7], on the other hand, found an exactly similar solution to the Navier-Stokes's equations concerning stagnation point flow against a stretching surface. Ref. [8] investigated hydromagnetic free convective heat transfer over a stretching surface embedded in a porous medium when heat generation or absorption was present. Meanwhile, [9] addressed the movement of viscoelastic flow near the stagnation point.

One of the most influential research fields in modern applied mathematics is fluid flow studies in mechanics due to nonlinear stretching [10]. Apart from air or water, the majority of fluids used in different processes have nonlinear viscosities [11]. Ref. [12] discussed stretching cylinders with both active and passive-controlled nanoparticle concentration conditions. They observed that the behavior of fluid momentum changes in the case of stretching cylinders and sheets was due to the nanoparticles' concentration. Ref. [13] studied heat transfer in the presence of hybrid nanofluid and found that these types of fluid transfer heat more effectively than conventional fluids. Ref. [13] unveiled the influence of the shapes of $\mathrm{Al}_{2} \mathrm{O}_{3}$ nanoparticles in an aqueous medium flowing in the porous space between eccentric cylinders. Ref. [14] studied finite surfaces with localized necking as bifurcation from deformation with wave numbers. They built an approach and validated that the necking problem is treated as a bifurcation with zero wave numbers. Ref. [15] analyzed the MHD flow of a Casson fluid over a stretching cylinder numerically. They revealed the reverse behavior of velocity and thermal fields by increasing the Hartman number. Nonlinear liquid connections are useful for understanding the flow behavior of commonly found liquids in nature and industry. As a result, various liquid models have been proposed to account for the popular characteristics of nonlinear liquids. Considerable attention towards convective flow and heat transfer in has been seen in recent years due to their nature and potential engineering applications. Ref. [16] examined two-dimensional flow with non-uniform heat generation with slip conditions. It was observed that an increase of the Eckert number also increased the thermal boundary layer and temperature distribution. The available literature on convective flow over stretching surfaces shows that the combined effects of nonlinear stretching, MHD, thermal radiation, and slip effects for distributions has not been investigated for Newtonian fluids.

Many researchers have published their findings on the significance of slip conditions in regards to the phenomenon of the lack of fluid adherence to surface borders. Slip has an important impact on the thickness and momentum of the thermal boundary layer. Furthermore, partial slip conditions are necessary in some practical circumstances such as fluid flow in micro-electro-mechanical devices. Some researchers have published their findings on the significance of slip states. Ref. [17] investigated heat transfer under slip conditions and found that as the Brownian motion and thermophoresis parameters increased, so did the local Nusselt number. Ref. [18] had obtained an exact solution by considering the effect of a second slip on nanofluids' peristaltic flow in an asymmetric channel. Ref. [19] had examined Hall current, Newtonian heating, and second-order slip effects on convective magneto-micropolar fluid flow over a sheet. Furthermore, increases in the slip decreased the horizontal velocity profile. Ref. [20] investigated second-order slip and heat transfer models over permeable stretching/shrinking surfaces. They discovered that there are multiple solutions for certain combinations of parameters, with an increase in slip value in the case of shrinking sheets. Ref. [21] investigated dual and triple solutions for an electrically conducting MHD slip flow and a non-Newtonian fluid past a shrinking sheet.

In natural flows, the MHD effects can be described by Navier-Stokes's equations for fluids. These equations describe the motion of a conducting fluid flowing under an external magnetic field's influence and, therefore, are of much importance. Ref. [22] investigated the steady laminar MHD mixed convection stagnation-point flow of an incompressible 
viscous fluid. They found that dual solutions exist in the presence of external magnetic fields both for assisting and opposing flows. Ref. [23] performed analyses for that heat and mass transfer for MHD viscoelastic flow with Ohmic dissipation. He had found that the magnetic parameter reduces heat transfer efficiency. Ref. [24] had studied second-grade MHD fluid in a porous medium with vorticity and Hall current effects. They concluded that the Newtonian flow field could be recovered by setting second-grade parameters equal to zero. Ref. [25] investigated the MHD flow of a micropolar liquid towards a nonlinear stretched surface using viscous dissipation, Joule heating, and a convective boundary state. They analyzed local Nusselt number enhances for the micropolar parameter, Biot, and Prandtl number. Ref. [26] used Buongiorno's model with nanoparticles for heat and mass transfer in the presence of MHD flow. They proposed using a passive control model to control the thickness of the boundary layer and the rate of mass transfer at the wall. The Buongiorno model was used to investigate MHD non-Newtonian Maxwell fluid with nanomaterials in an exponentially stretching surface [27]. They discovered that the thermophoresis parameter has a negligible effect on the temperature profile. Because of the curved stretching sheet, researchers investigated mass and heat transfer in MHD flow of an electrically conducting non-Newtonian micropolar fluid. Ref. [28] discovered that magnetic field interaction increased fluid temperature while decreasing microrotation and velocities at all domain flow points. Ref. [29] studied the thermal analysis of radiative bioconvection MHD flow comprising gyrotactic microorganism with activation energy. They concluded the density of motile microorganisms decreases with Peclet number and bioconvection Lewis number.

The current study investigates nonlinear extended flow for MHD viscous fluid flow over a cylinder in conjunction with nonlinear thermal radiations and slips effects for both velocity and temperature distributions. Therefore, an attempt is made to investigate the resulting nonlinear boundary layer problem numerically. The governing partial differential equations of the physical model have been reduced to ordinary differential equations (ODEs). The Runge-Kutta-Fehlberg method, in conjunction with shooting procedures, was used to numerically solve the resulting system of equations. Furthermore, graphical findings are shown and analyzed.

\section{Mathematical Formulation}

The behavior of two-dimensional incompressible boundary layer Newtonian fluid flow is apprehended in this article. In two-dimensional cylindrical flow, the radius of the cylinder is greater than the thickness of the boundary layer [30]. The following conditions were applied and considered from the applications point of view (i.e., MHD): velocitytemperature slips, nonlinear thermal radiations, and stretching. Nonlinear stretching cylinder of radius $\mathrm{R}$ in the presence of a non-uniform magnetic field $B=B_{0}(z)^{\frac{n-1}{2}}$. The flow is confined in the region $r>R$, where $z$ is the horizontal axis of the cylindrical coordinate system (and is taken along the cylinder's axis) while $r$ and $\theta$ are radial and tangential components, respectively. The flow is generated by stretching the cylinder under the influence of two forces equal in magnitude and opposite in direction along the $z$-axis, keeping the origin fixed. We suppose that at the time, $t<0$, the fluid is at rest. The cylinder is stretched with the velocity $U(z)=U_{0} z^{n}$, where $U_{0}$ and $n$ are constant.

The assumed nonlinear temperature $T_{w}(z)=T_{\infty}+b z^{2 n-1}$ function of $z$. Free stream velocity is $u_{e}=u_{0} z^{n}$ where $U_{0}>>u_{0}$ ambient fluid temperature $T_{\infty}$, thermal diffusivity $\alpha$, thermal expansion coefficient $\beta$, and the magnetic field are applied traversal to the cylinder's axis. Flow description and coordinate axis are shown in Figure 1. 


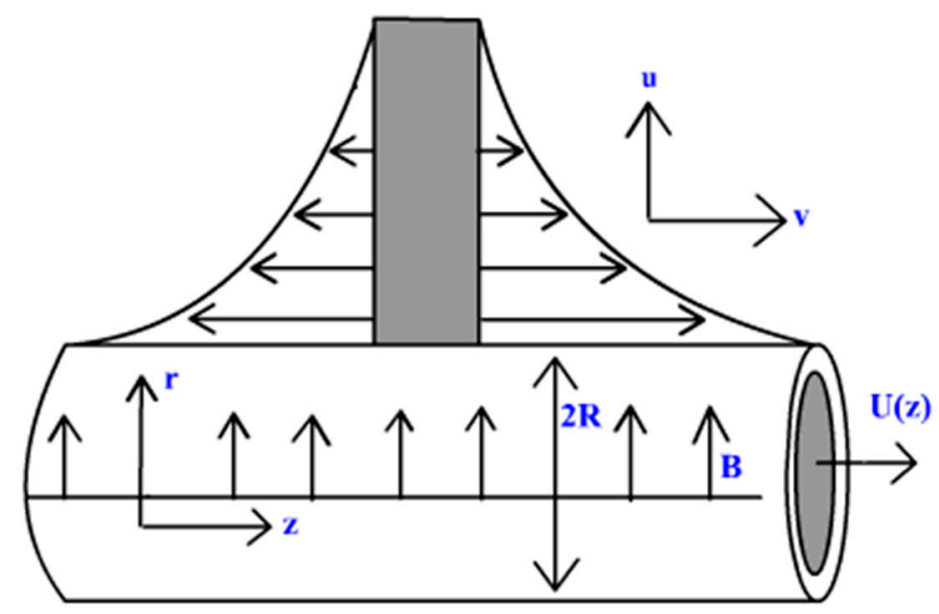

Figure 1. Geometrical concept of the physical model.

The boundary layer's assumptions are taken as mentioned [15,31,32]. Governing flow and heat transfer equations with the Boussinesq approximation are:

$$
\begin{gathered}
\frac{\partial(r u)}{\partial z}+\frac{\partial(r v)}{\partial r}=0 \\
u \frac{\partial u}{\partial z}+v \frac{\partial u}{\partial r}=u_{e} \frac{d u_{e}}{d z}+\frac{v}{r} \frac{\partial}{\partial r}\left(r \frac{\partial u}{\partial r}\right)-\frac{\sigma B^{2}}{\rho} u+g \beta\left(T-T_{\infty}\right), \\
u \frac{\partial T}{\partial z}+v \frac{\partial T}{\partial r}=\frac{\alpha}{r} \frac{\partial}{\partial r}\left(r \frac{\partial T}{\partial r}\right)-\frac{1}{\rho c_{p}} \frac{\partial q_{r}}{\partial r}
\end{gathered}
$$

With corresponding boundary conditions $(t=0)$,

$$
\left.\begin{array}{l}
u=U(z)+L \frac{\partial u}{\partial r}, v=0, T=T_{w}(z)+S \frac{\partial T}{\partial r} \text { at } r=R, \\
u \rightarrow u_{e}, T \rightarrow T_{\infty} \text { as } r \rightarrow \infty,
\end{array}\right\}
$$

Velocity slip $L$, thermal slip length $S$, and stretching index $n$ are constants. The supposed temperature difference within the BLF is such that $T^{4}$ may be expanded in Taylor's series about $T_{\infty}$ and neglect the higher terms. Thus, we get,

$$
T^{4} \approx 4 T_{\infty}^{3} T-3 T_{\infty}^{4}
$$

and

$$
q_{r} \approx-\frac{4 \sigma^{*}}{3 k^{*}} \frac{\partial T^{4}}{\partial r} .
$$

By substituting Equation (5) in Equation (6) we acquired [33],

$$
q_{r} \approx-\frac{16 \sigma^{*} T_{\infty}^{3}}{3 k^{*}} \frac{\partial T}{\partial r} .
$$

Here, the mean absorption coefficient $k^{*}$, Stefan-Boltzmann constant $\sigma^{*}$ and Quinn Brewster heat flux $q_{r}$ given by [34] using Rosseland approximation.

The following similarity transformations are given for the similar solution:

$$
\eta=\frac{r^{2}-R^{2}}{2 R}\left(\frac{U}{v z}\right)^{\frac{1}{2}}, \psi=(U v z)^{\frac{1}{2}} R f(\eta), \theta(\eta)=\frac{T-T_{\infty}}{T_{w}-T_{\infty}} .
$$


After using similarity transformation, Equations (2)-(4) become Equations (9)-(11). Equation (1) is satisfied identically,

$$
\begin{gathered}
(1+2 \eta C) f^{\prime \prime \prime}+2 C f^{\prime \prime}+\left(\frac{n+1}{2}\right) f f^{\prime \prime}-M f^{\prime}-n f^{\prime 2}+\lambda \theta=0, \\
\left(1+\frac{4}{3} K\right)(1+2 \eta C) \theta^{\prime \prime}+\left(2+\frac{4}{3} K\right) C \theta^{\prime}+\frac{\operatorname{Pr}}{2}\left[(n+1) f \theta^{\prime}-(4 n-2) \theta f^{\prime}\right]=0,
\end{gathered}
$$

boundary conditions,

$$
\left.\begin{array}{cc}
f^{\prime}=1+\delta f^{\prime \prime}, f=0, \theta=1+\gamma \theta^{\prime} & \text { at } \eta=0 \\
f^{\prime} \rightarrow 0, \theta \rightarrow 0 & \text { as } \eta \rightarrow \infty
\end{array}\right\}
$$

In the above equations, prime denotes the derivative for the independent similarity variable is $\eta$, dimensionless temperature $\theta$, stream function $\psi$, velocity slip $\delta=L\left(\frac{u}{v z}\right)^{\frac{1}{2}}$, temperature slip $\gamma=S\left(\frac{U}{v z}\right)^{\frac{1}{2}}$, mixed convection parameter $\lambda=\frac{g \beta b}{U_{0}^{2}}$, magnetic parameter $M=\left(\frac{\sigma B_{0}^{2}}{\rho U_{0}}\right)^{\frac{1}{2}}$, curvature parameter $C=\frac{2 \eta v}{r^{2}-R^{2}}\left(\frac{z}{U}\right)$, radiation parameter $K=\frac{4 \sigma^{*} T_{*}^{3}}{k k^{*}}$, and Prandtl number $\operatorname{Pr}=\frac{v}{\alpha}$. It is worth mentioning that assisting flow is $\lambda>0$, opposing flow $\lambda<0$, and forced convection flow $\lambda=0$. Industrial interest quantities are local skin friction, $C_{f}$ and local Nusselt number, $N u$. These are directly proportional to $f^{\prime \prime}(0)$ and $-\theta^{\prime}(0)$, respectively.

$$
C_{f}=\frac{2 \tau_{w}}{\rho U^{2}}, N u=\frac{x q_{w}}{k\left(T_{w}-T_{\infty}\right)}
$$

Here, surface shear stress and heat flux of the stretching cylinder is denoted by $\tau_{w}$ and $q_{w}$, respectively. Applying the similarity transformation on Equations (12) and (13) achieves,

$$
-\theta^{\prime}(0)=R e_{z}^{-\frac{1}{2}} N u, f^{\prime \prime}(0)=\frac{1}{4} R e_{z}^{\frac{1}{2}} C_{f}
$$

\section{Numerical Method}

The present model contains the nonlinear dimensionless coupled ODEs in Equations (9)-(13) with sufficient boundary conditions. Both Runge-Kutta-Fehlberg's fourthand fifth-order (RKF45) and the shooting scheme were used to solve the ODEs [13,35]. To solve these coupled nonlinear differential equations, we converted higher-order nonlinear ODEs into first-order ODEs. Following that, the applicable initial solutions (zeroth order) were chosen, which must validate the boundary conditions under consideration. The obtained solutions demonstrated that a variety of relevant parameters affect velocity and temperature distributions. The shooting approach was repeated in order to achieve the convergence criteria so that no numerical oscillations would occur. The $\Delta \eta=0.01$ step size was taken, and the selection $\eta_{\infty}$ varied from 3 to 5 .

$$
\left.\begin{array}{rl}
f^{\prime} & =x, \\
x^{\prime} & =y, \\
y^{\prime} & =\frac{n x^{2}+M x-\left(\frac{n+1}{2}\right) f y-2 C y-\lambda \theta}{(1+2 \eta C)},
\end{array}\right\}
$$

boundary conditions,

$$
f(0)=0, f^{\prime}(0)=1+\delta f^{\prime \prime}(0), \theta(0)=1+\gamma \theta^{\prime} .
$$




\section{Model Validity}

It is significant to validate and confirm the accuracy of the present numerical procedure. This model's validity is confirmed by comparing the special case with previous studies in the literature. In reference to other studies, our results agreed well with them (as mentioned in Tables 1 and 2). The present results of the local Nusselt number in the Newtonian fluid, compared with the existing data for different temperature exponent values, are present in Table 1. The skin friction coefficient numeric errors are also considered in Table 2. An agreement was found between both physical phenomena and the publicized data.

Table 1. Comparison between present and previous encountered results of $\theta^{\prime}(0)$.

\begin{tabular}{ccccc}
\hline $\boldsymbol{n}$ & {$[36]$} & {$[37]$} & {$[30]$} & Present \\
\hline 0 & 0.5820 & 0.5820 & 0.5821 & 0.5820 \\
\hline 1 & 1.0000 & 1.0000 & 1.0000 & 0.9961 \\
\hline 2 & 1.3333 & 1.3333 & 1.3332 & 1.3333 \\
\hline
\end{tabular}

Table 2. Error in $f^{\prime \prime}(0)$ values carried out between analytic and numeric solutions of at plate with the no-slip condition for $n=1, \operatorname{Pr}=1$.

\begin{tabular}{cccc}
\hline $\boldsymbol{M}$ & Analytical Solution & Numerical Solution & Error \\
\hline 0 & -1.00000 & -1.00001 & -0.00001 \\
\hline 0.5 & -1.11803 & -1.11803 & -0.00000 \\
\hline 1.0 & -1.41421 & -1.41421 & -0.00000 \\
\hline 1.5 & -1.80277 & -1.80278 & -0.00001 \\
\hline
\end{tabular}

\section{Results and Discussions}

The non-linearity parameter $n$ for the velocity profile has been taken into account in Figure 2 . Here, the velocity profile decreased when the fluid exhibited heavy oscillating behavior. Furthermore, for greater values of $n$, the velocity profile became zero in the short-range.

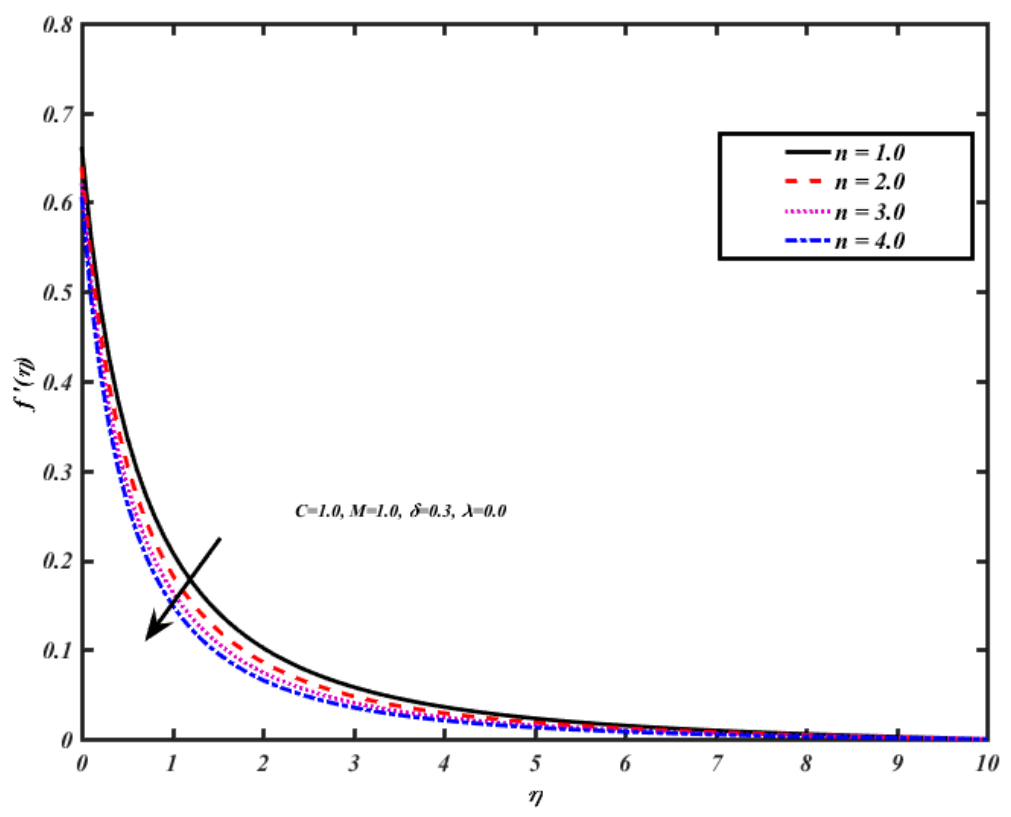

Figure 2. Illustration of $f^{\prime}(\eta)$ for different values of $n$. 
In Figure 3, an increase in the curvature made the cylinder cross-section smaller. It enhanced the fluid velocity due to the small surface area. A smaller surface means a smaller amount of friction production between the solid and liquid surface.

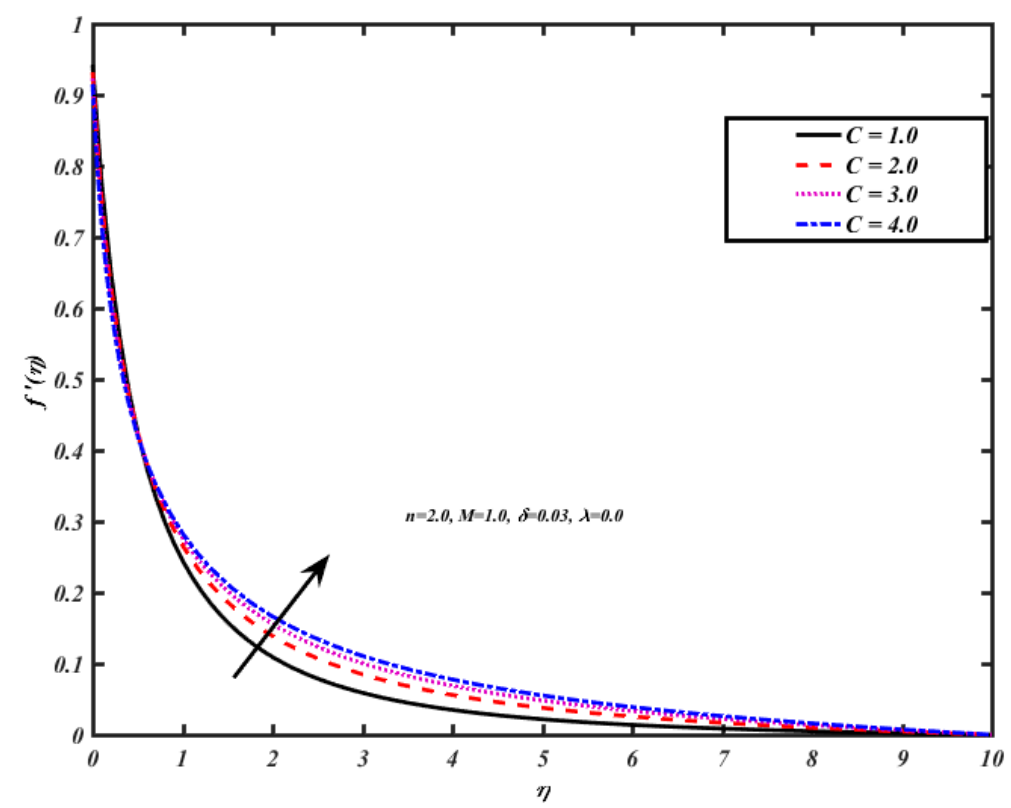

Figure 3. Illustration of for different values of $f^{\prime}(\eta)$ for different values of $C$.

Figure 4 elucidates the magnetic field influence on the velocity profile. Due to this field, Lorentz force generation started. It came into action in order to oppose the momentum of the electrical conducting fluid.

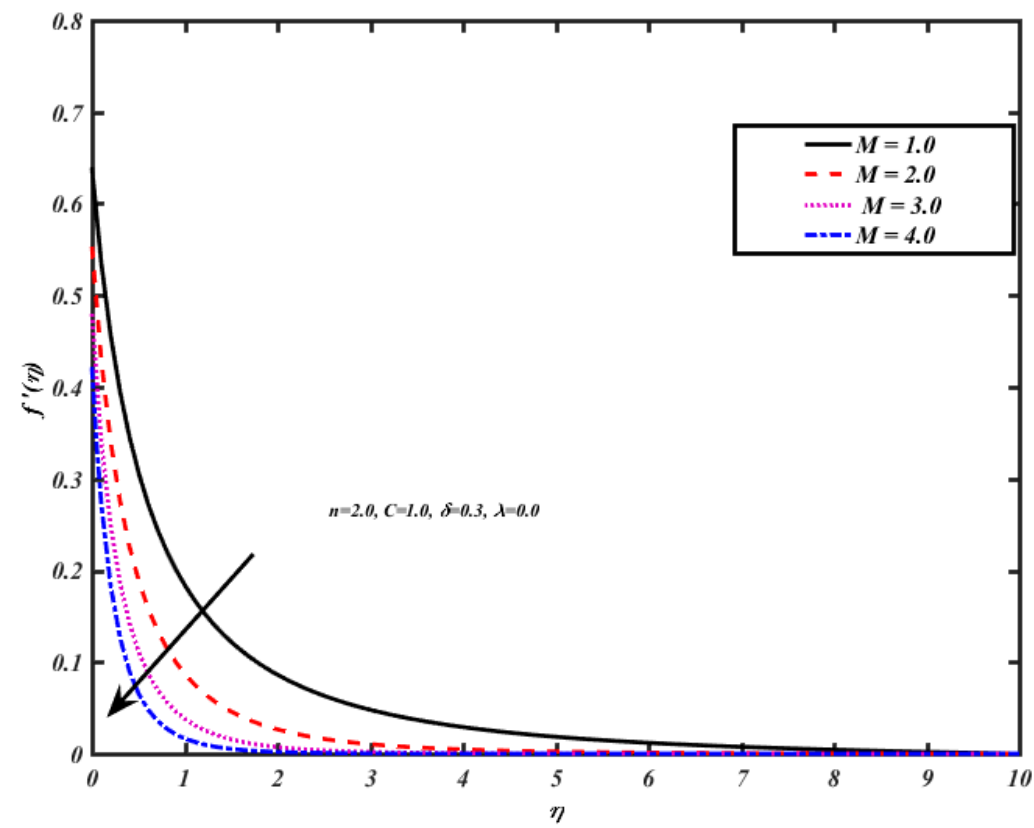

Figure 4. Illustration of $f^{\prime}(\eta)$ for different values of $M$.

Figure 5 represents the velocity-slip effect on the velocity profile. The abrupt decrease in the momentum boundary layer was due to this effect. Here, the measured viscosity was significantly lower than the actual viscosity of the fluid. 


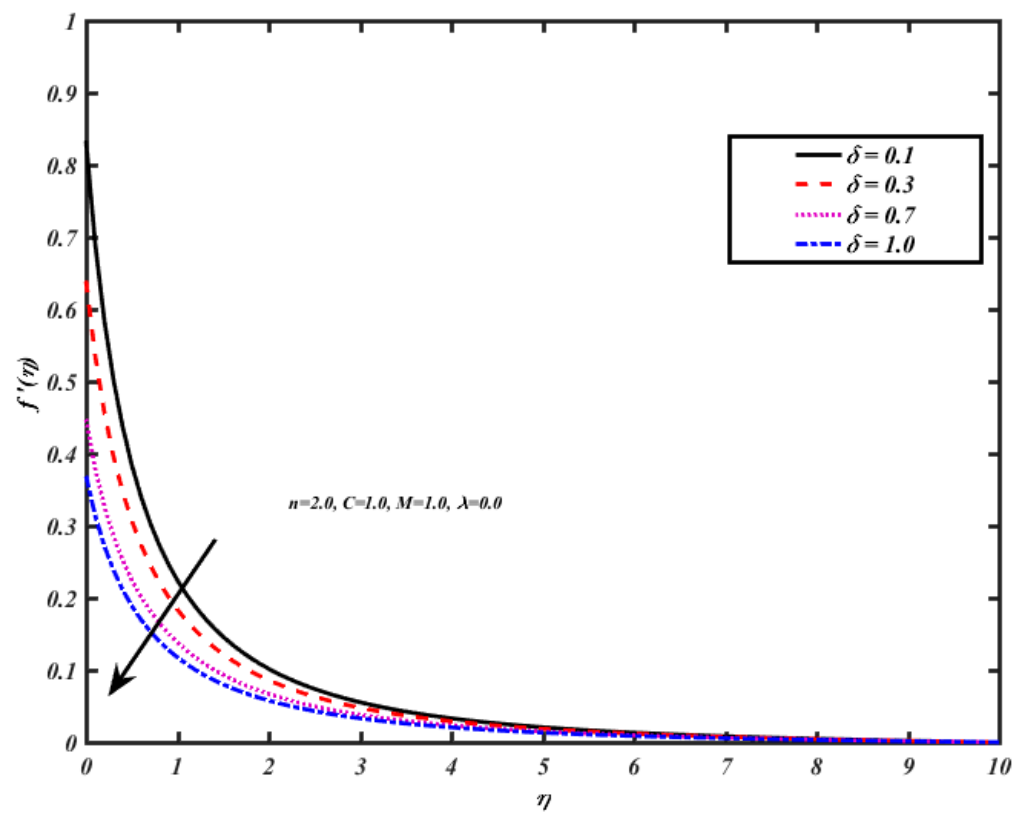

Figure 5. Illustration of $f^{\prime}(\eta)$ for different values of $\delta$.

In Figure 6, the velocity profile is portrayed in order to measure the influence of mixed convection. According to this graph, it was observed that the velocity profile was enhanced with an increase in the value of the mixed convection effect. This was because an increase in buoyancy caused an increase in the momentum of the fluid.

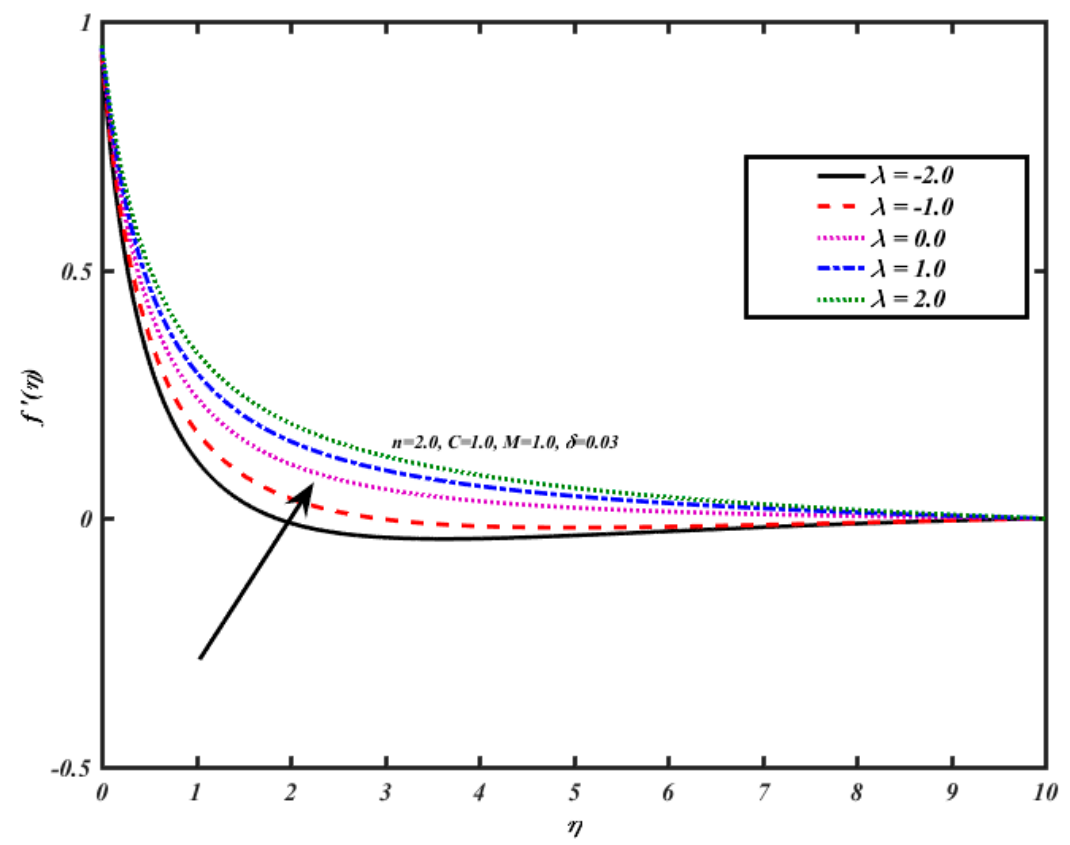

Figure 6. Illustration of $f^{\prime}(\eta)$ for different values of $\lambda$.

Figure 7 shows the effect of the non-linearity parameter on the temperature profile. It reveals that an increment in $n$ reduced the temperature profile. Nonlinear stretching caused a disturbance in the flow, which caused the excess particles of fluid to collide with each other. 


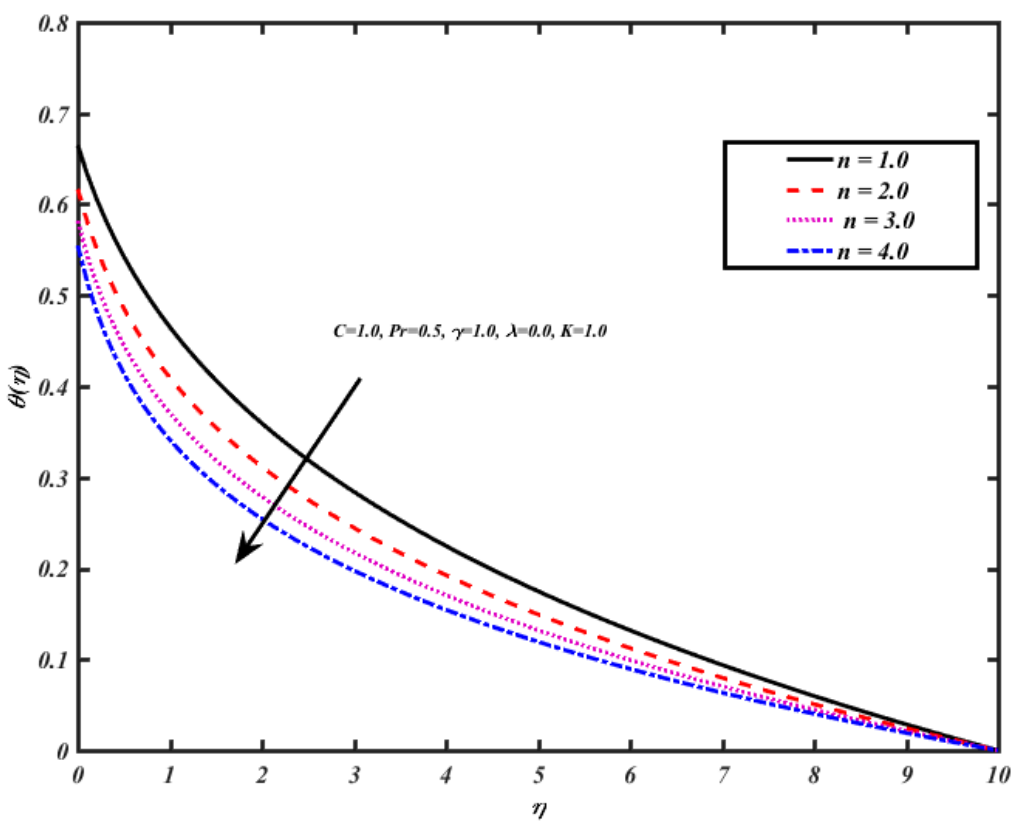

Figure 7. Illustration of $\theta(\eta)$ for different values of $n$.

Figure 8 highlights the curvature parameter variation. An increase in the values of the curvature parameter enhanced the momentum boundary layer (as mentioned in Figure 2). This caused the fluid to move in faster and have less interaction or collision with the fluid molecules. Since there was less collision between the molecules, the small amount of friction generated a lesser amount of heat. This reduced the overall temperature profile.

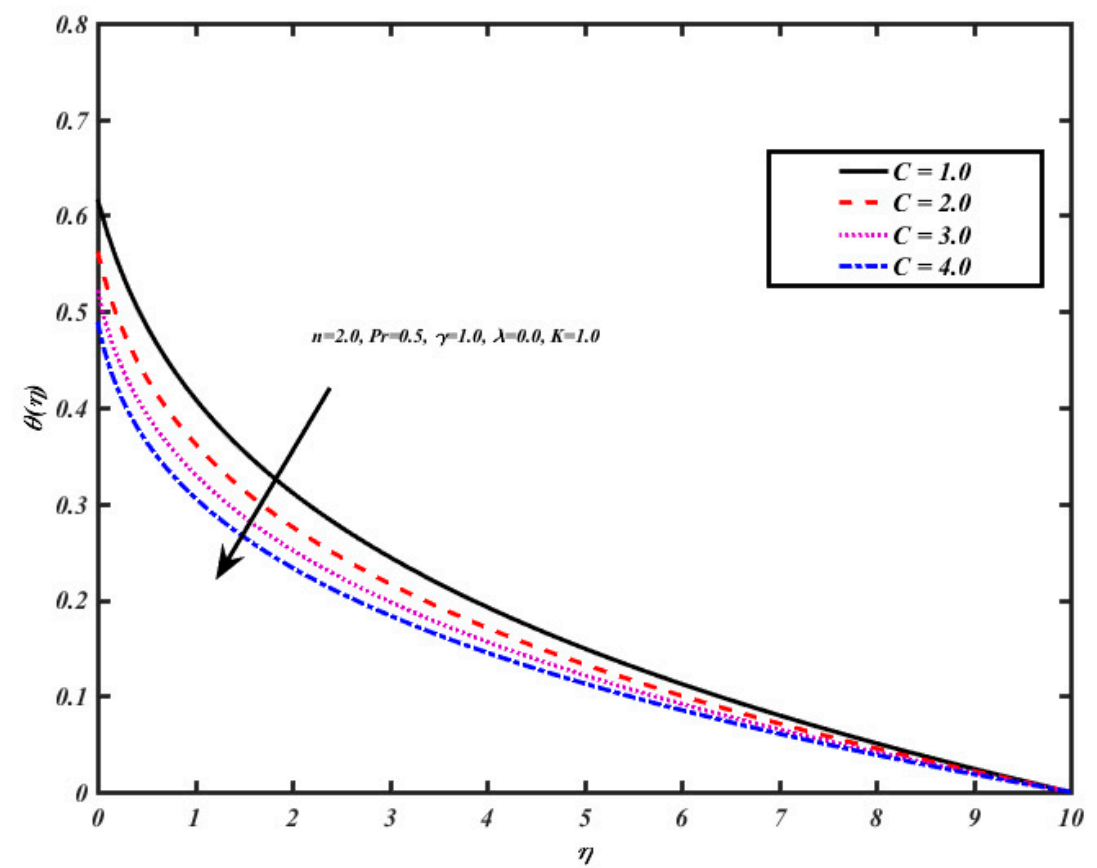

Figure 8. Illustration of $\theta(\eta)$ for different values of $C$.

The effect of the Prandtl number on the temperature profile is shown in Figure 9. The Prandtl number is the ratio of kinematic viscosity to thermal diffusivity that varies with temperature. Owing to this fact, different fluids can have different Prandtl numbers because of variations in their temperature. In this case, the fundamental law is that fulfilling a smaller value of Pr decreases the temperature profile. 


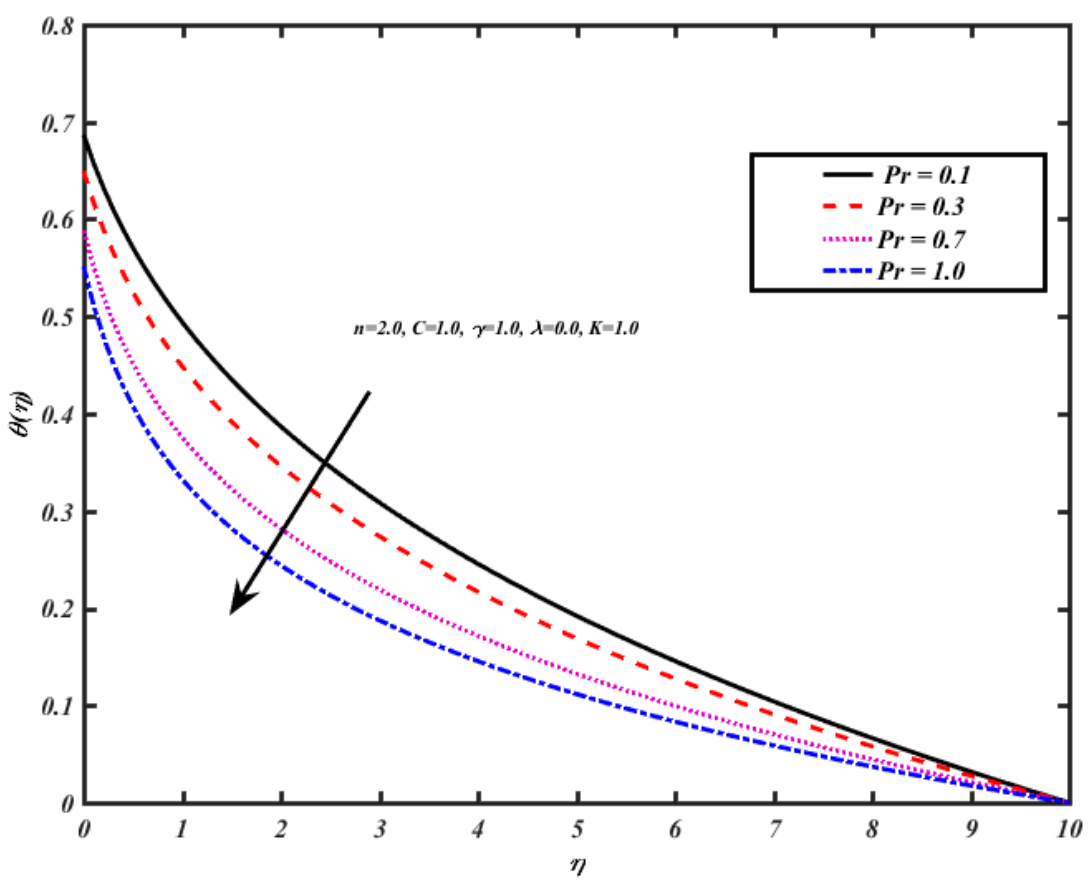

Figure 9. Illustration of $\theta(\eta)$ for different values of Pr.

The temperature profile, as shown in Figure 10, decreased as the value of the thermal slip increased. As the temperature rose, the heat transfer from the solid surface to the fluid decreased. As a result, the temperature boundary layer decreased. Furthermore, although the momentum equation is dependent on $\theta(\eta)$, no important effect $\gamma$ on velocity profiles was observed.

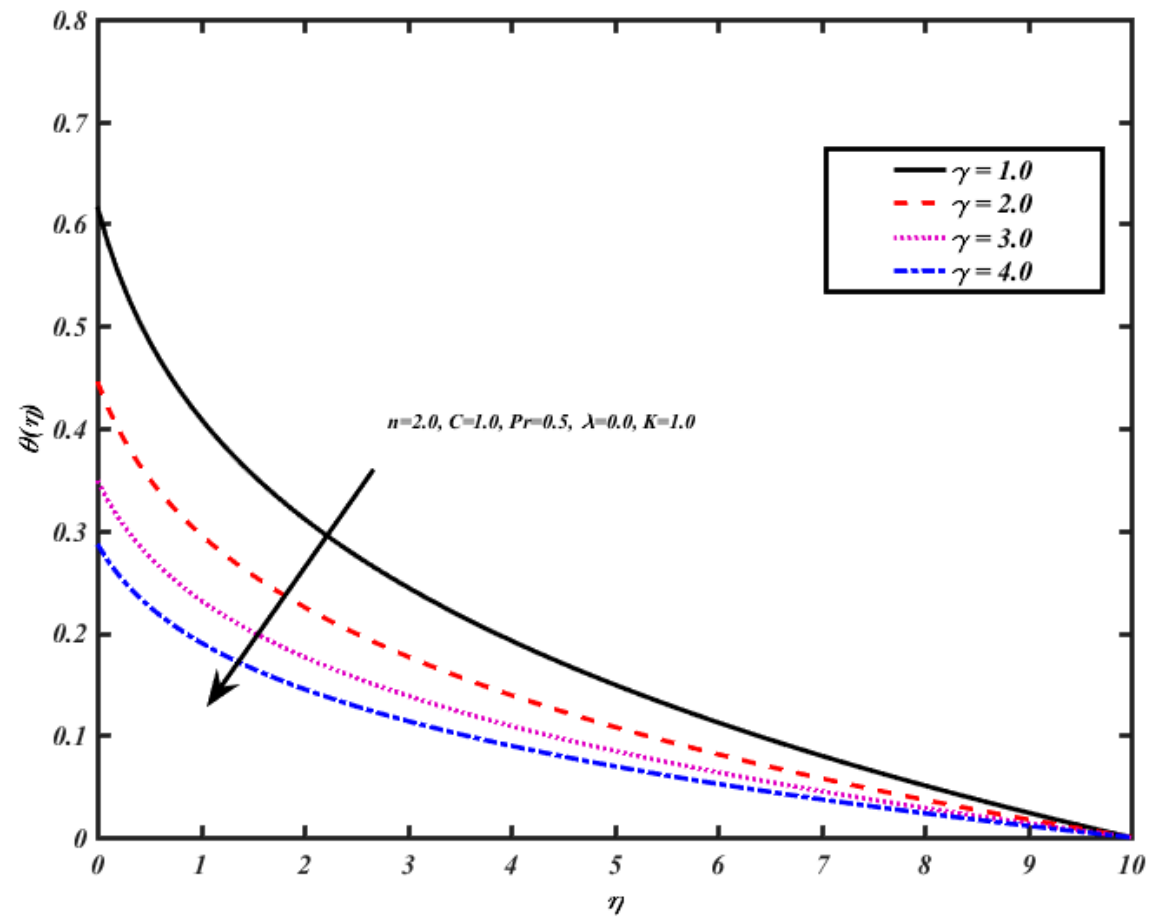

Figure 10. Illustration of $\theta(\eta)$ for different values of $\gamma$.

Figure 11 exhibits that temperature profiles decreased rapidly with the increment in mixed convection. It had a prominent effect due to coupled governing equations. In general, the mixed convective heat transfer phenomena, due to fluid motion, occurs when 
the heated fluid moves away from the heat source or heated surface. It makes the fluid have a lower temperature near the stretch surface, reducing the overall temperature profile.

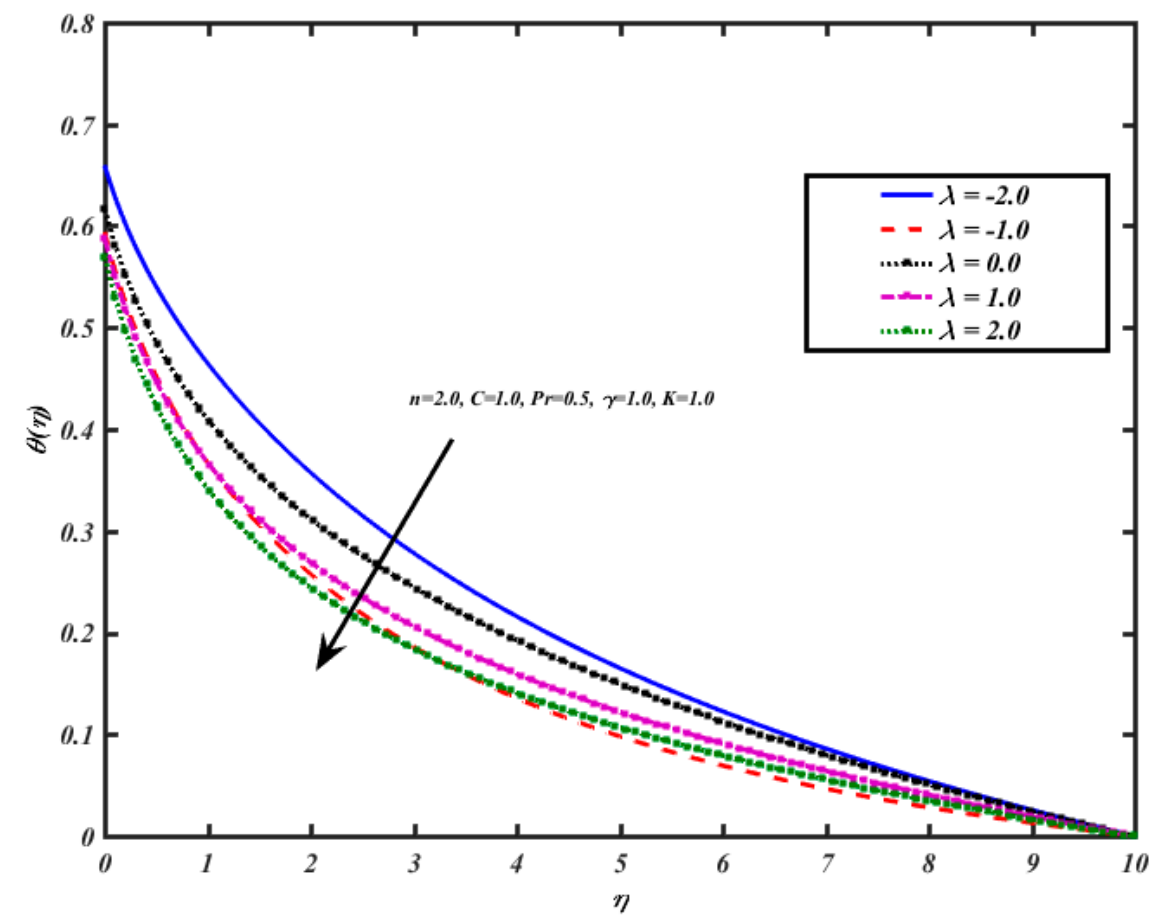

Figure 11. Illustration of $\theta(\eta)$ for different values of $\lambda$.

Figure 12 shows the thermal radiations effect on the temperature profile. It was concluded that an increase in $K$ also increased the temperature profile. It is a common fact that, in order to enhance thermal radiation, the temperature of the surrounding environment must also rise. Therefore, in this case, an excess amount of radiation enhanced the temperature and boundary thickness.

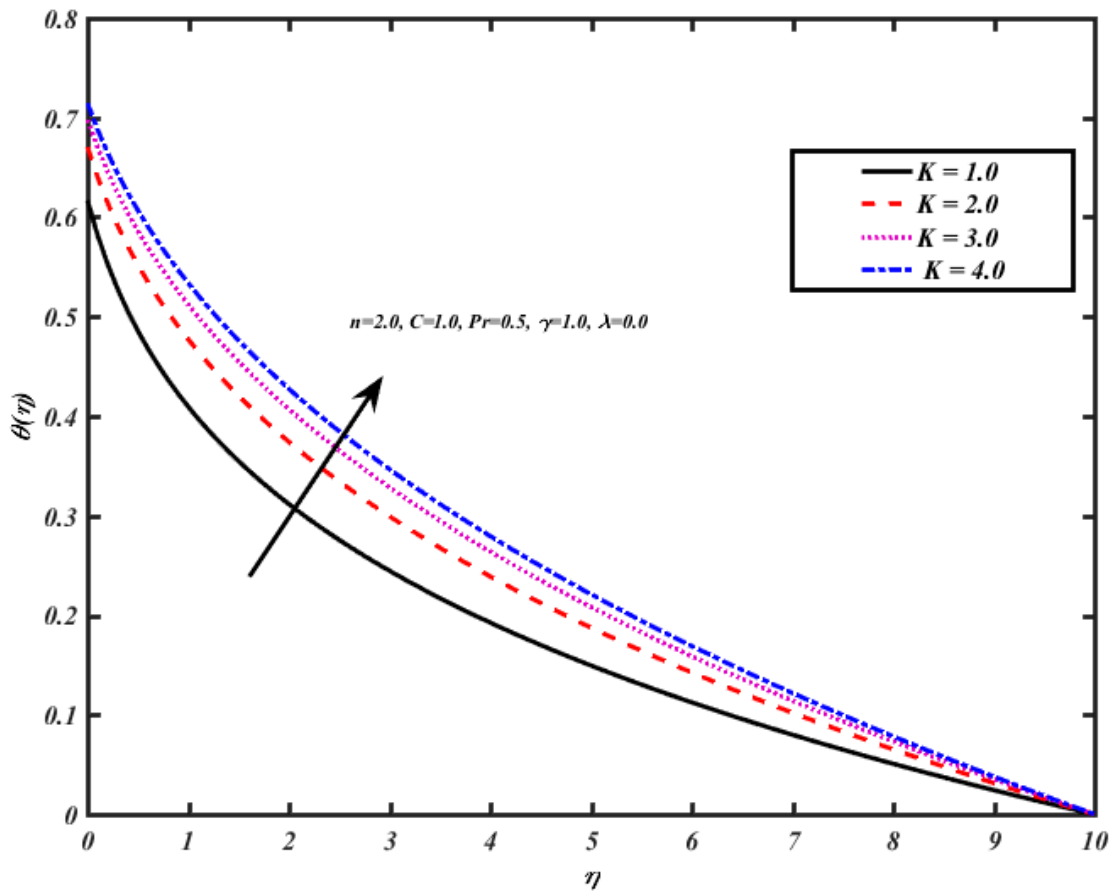

Figure 12. Illustration of $\theta(\eta)$ for different values of $K$. 
In Table 3 , the skin friction $-f^{\prime \prime}(0)$ coefficient showed decreasing behavior for the non-linearity exponent, curvature parameter, and magnetic parameter. The Nusselt number $-\theta^{\prime}(0)$ showed decreasing behavior for the non-linearity exponent, curvature parameter, mixed convection parameter, and Prandtl number.

Table 3. Comparison table of skin friction and Nusselt number

\begin{tabular}{|c|c|c|c|c|c|c|c|c|c|}
\hline$n$ & $C$ & $M$ & $\delta$ & $\lambda$ & $\operatorname{Pr}$ & $\gamma$ & $K$ & $-f^{\prime \prime}(0)$ & $-\theta^{\prime}(0)$ \\
\hline 1 & & & & & & & & 1.12685 & 0.33489 \\
\hline 2 & & & & & & & & 1.20155 & 0.38309 \\
\hline 3 & & & & & & & & 1.26145 & 0.41789 \\
\hline \multirow[t]{21}{*}{2} & 1 & & & & & & & 1.20155 & 0.38309 \\
\hline & 2 & & & & & & & 1.33150 & 0.43739 \\
\hline & 3 & & & & & & & 1.44063 & 0.47790 \\
\hline & 1 & 1 & & & & & & 1.20155 & 0.38309 \\
\hline & & 2 & & & & & & 1.48689 & 0.35397 \\
\hline & & 3 & & & & & & 1.73211 & 0.33479 \\
\hline & & 1 & 0.1 & & & & & 1.66038 & 0.40184 \\
\hline & & & 0.5 & & & & & 0.94980 & 0.37065 \\
\hline & & & 1 & & & & & 0.63069 & 0.35164 \\
\hline & & & 0.3 & -2 & & & & 1.71442 & 0.34021 \\
\hline & & & & 0 & & & & 1.20155 & 0.38309 \\
\hline & & & & 2 & & & & 0.83701 & 0.43027 \\
\hline & & & & 0 & 0.1 & & & 1.20155 & 0.31291 \\
\hline & & & & & 0.5 & & & 0.83701 & 0.38309 \\
\hline & & & & & 1 & & & 1.20155 & 0.44830 \\
\hline & & & & & 0.5 & 1 & & 1.20155 & 0.38309 \\
\hline & & & & & & 2 & & 1.20155 & 0.27698 \\
\hline & & & & & & 3 & & 1.20155 & 0.21691 \\
\hline & & & & & & 1 & 1 & 1.20155 & 0.38309 \\
\hline & & & & & & & 2 & 1.20155 & 0.32934 \\
\hline & & & & & & & 3 & 1.20155 & 0.30183 \\
\hline
\end{tabular}

\section{Conclusions}

In this study, we investigated the effects of velocity-temperature slip on steady flow and heat transfer over an axisymmetric cylinder with mixed convection. We also investigated both the traversal magnetic field and thermal radiation with non-linearity exponent behavior. By using the appropriate similarity transformations, the governing equations were reduced to ODEs. The shooting technique was used to reduce the ODEs and was solved numerically through RKF45. Graphs and tables were used to present and discuss the effect of main parameters on velocity and temperature profiles, as well as on the skin friction coefficient and Nusselt number. A stability analysis was also performed for the accuracy and validation of the results, and found that the present results are in good agreement with previous studies. In this work, we showed that:

- $\quad$ The mixed convection parameter enhances the velocity profile.

- The Prandtl number reduces the temperature distribution across the flow.

- The velocity profile abruptly decreases in the presence of a velocity slip.

- The temperature profile diminishes due to the thermal slip effect. 
- The skin friction was observed to increase for larger values of velocity slip and mixed convection parameter.

- The Nusselt number increased due to an increase of magnetic, velocity-temperature slip, and radiation parameters.

Author Contributions: Conceptualization, M.T.; Funding acquisition, J.R.L. and D.Y.S.; Methodology, M.T.; Project administration, M.K.; Software, M.T., A.F. and D.Y.S.; Supervision, M.K. and J.R.L.; Visualization, J.R.L.; Writing—original draft, S.R., A.F. and R.K.; Writing—review \& editing, S.R., A.F., R.K. and D.Y.S. All authors have read and agreed to the published version of the manuscript.

Funding: No funds are given for this work.

Institutional Review Board Statement: Not applicable.

Informed Consent Statement: Not applicable.

Data Availability Statement: Not applicable.

Conflicts of Interest: The authors declare no conflict of interest.

\section{Nomenclature}

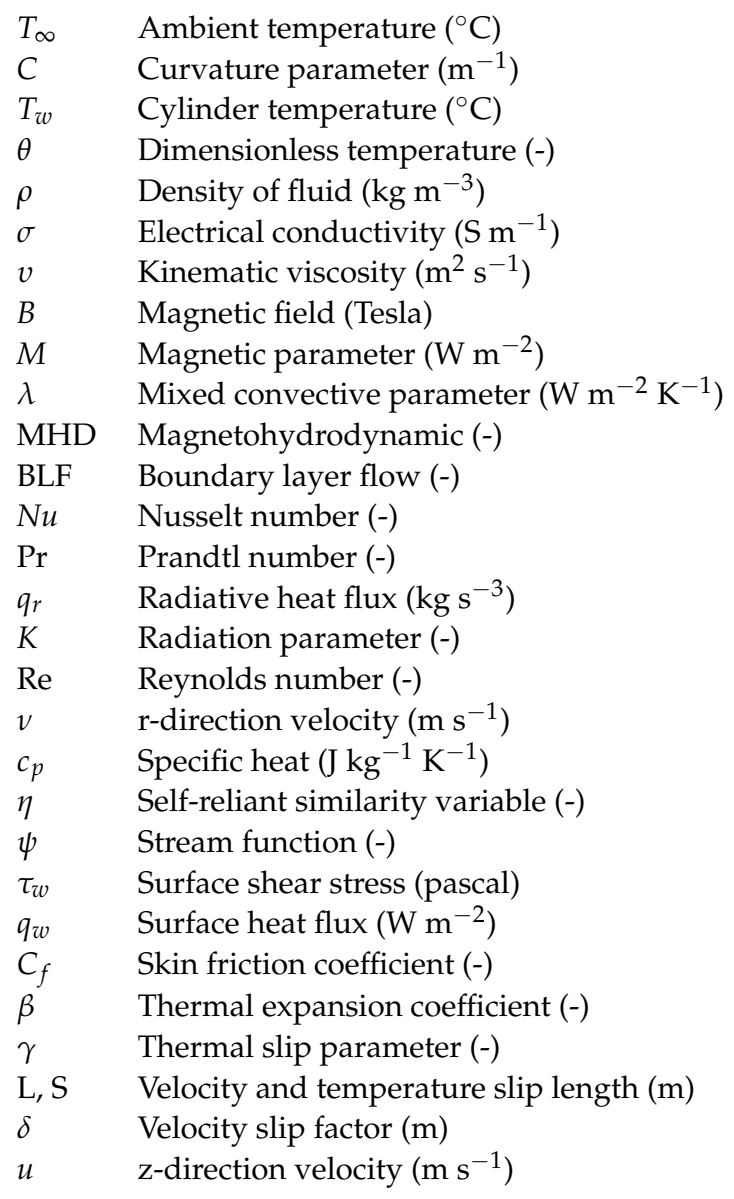

\section{References}

1. Abbasi, A.; Mabood, F.; Farooq, W.; Hussain, Z. Non-orthogonal stagnation point flow of Maxwell nano-material over a stretching cylinder. Int. Commun. Heat Mass Transf. 2021, 120, 105043. [CrossRef]

2. Abo-Eldahab, E.M.; El Aziz, M.A. Hydromagnetic three-dimensional free convective heat transfer overa stretching surface embedded in a non-Darcian porous medium inthe presence of heat generation or absorption. Can. J. Phys. 2005, 83, 739-751. [CrossRef] 
3. Akbar, T.; Nawaz, R.; Kamran, M.; Rasheed, A. Magnetohydrodynamic (MHD) flow analysis of second grade fluids in a porous medium with prescribed vorticity. AIP Adv. 2015, 5, 117133. [CrossRef]

4. Ali, F.M.; Nazar, R.; Arifin, N.M.; Pop, I. Mixed convection stagnation-point flow on vertical stretching sheet with external magnetic field. Appl. Math. Mech. 2014, 35, 155-166. [CrossRef]

5. Aly, E.H.; Ebaid, A. Effect of the velocity second slip boundary condition on the peristaltic flow of nanofluids in an asymmetric channel: Exact solution. Abstr. Appl. Anal. 2014, 2014, 191876. [CrossRef]

6. Andersson, H.I. Slip flow past a stretching surface. Acta Mech. 2002, 158, 121-125. [CrossRef]

7. Arifuzzaman, S.M.; Khan, M.; Mehedi, M.F.; Rana, B.; Ahmmed, S. Chemically reactive and naturally convective high speed MHD fluid flow through an oscillatory vertical porous plate with heat and radiation absorption effect. Eng. Sci. Technol. Int. J. 2018, 21, 215-228. [CrossRef]

8. Brady, J.F.; Acrivos, A. Steady flow in a channel or tube with an accelerating surface velocity. An exact solution to the NavierStokes equations with reverse flow. J. Fluid Mech. 1981, 112, 127-150. [CrossRef]

9. Brewster, M.Q. Thermal Radiative Transfer and Properties; John Wiley \& Sons: Hoboken, NJ, USA, 1992.

10. Crane, L.J. Flow past a stretching plate. Z. Angew. Math. Phys. ZAMP 1970, 21, 645-647. [CrossRef]

11. Farooq, U.; Lu, D.; Munir, S.; Ramzan, M.; Suleman, M.; Hussain, S. MHD flow of Maxwell fluid with nanomaterials due to an exponentially stretching surface. Sci. Rep. 2019, 9, 7312. [CrossRef]

12. Ferdows, M.; Khan, M.S.; Alam, M.M.; Sun, S. MHD mixed convective boundary layer flow of a nanofluid through a porous medium due to an exponentially stretching sheet. Math. Probl. Eng. 2012, 2012, 408528. [CrossRef]

13. Hayat, T.; Tamoor, M.; Khan, M.I.; Alsaedi, A. Numerical simulation for nonlinear radiative flow by convective cylinder. Results Phys. 2016, 6, 1031-1035. [CrossRef]

14. Hsiao, K.-L. Heat and mass mixed convection for MHD visco-elastic fluid past a stretching sheet with ohmic dissipation. Commun. Nonlinear Sci. Numer. Simul. 2010, 15, 1803-1812. [CrossRef]

15. Ibrahim, W.; Shanker, B. MHD boundary layer flow and heat transfer of a nanofluid past a permeable stretching sheet with velocity, thermal and solutal slip boundary conditions. Comput. Fluids 2013, 75, 1-10. [CrossRef]

16. Kamran, M.; Wiwatanapataphee, B.; Vajravelu, K. Hall current, Newtonian heating and second-order slip effects on convective magneto-micropolar fluid flow over a sheet. Int. J. Mod. Phys. C 2018, 29, 1850090. [CrossRef]

17. Mahapatra, T.; Gupta, A. Stagnation point flow towards a stretching surface. Can. J. Chem. Eng. 2008, 81, 258-263. [CrossRef]

18. Mahapatra, T.R.; Gupta, A.S. Stagnation-Point flow of a viscoelastic fluid towards a stretching surface. Int. J. Non Linear Mech. 2004, 39, 811-820. [CrossRef]

19. Majeed, A.; Zeeshan, A.; Amin, N.; Ijaz, N.; Saeed, T. Thermal analysis of radiative bioconvection magnetohydrodynamic flow comprising gyrotactic microorganism with activation energy. J. Therm. Anal. Calorim. 2021, 143, 2545-2556. [CrossRef]

20. Majeed, A.; Zeeshan, A.; Noori, F.M.; Masud, U. Influence of rotating magnetic field on Maxwell saturated ferrofluid flow over a heated stretching sheet with heat generation/absorption. Mech. Ind. 2019, 20, 502. [CrossRef]

21. Maskeen, M.M.; Zeeshan, A.; Mehmood, O.U.; Hassan, M. Heat transfer enhancement in hydromagnetic alumina-copper/water hybrid nanofluid flow over a stretching cylinder. J. Therm. Anal. Calorim. 2019, 138, 1127-1136. [CrossRef]

22. Mohyud-Din, S.T.; Khan, U.; Ahmed, N.; Rashidi, M.M. A study of heat and mass transfer on magnetohydrodynamic (MHD) flow of nanoparticles. Propuls. Power Res. 2018, 7, 72-77. [CrossRef]

23. Mukhopadhyay, S. Effects of thermal radiation and variable fluid viscosity on stagnation point flow past a porous stretching sheet. Meccanica 2013, 48, 1717-1730. [CrossRef]

24. Mukhopadhyay, S. MHD boundary layer slip flow along a stretching cylinder. Ain Shams Eng. J. 2013, 4, 317-324. [CrossRef]

25. Mukhopadhyay, S.; De, P.R.; Bhattacharyya, K.; Layek, G.C. Casson fluid flow over an unsteady stretching surface. Ain Shams Eng. J. 2013, 4, 933-938. [CrossRef]

26. Rasheed, H.U.; Khan, Z.; Islam, S.; Khan, I.; Guirao, J.L.G.; Khan, W. Investigation of two-dimensional viscoelastic fluid with nonuniform heat generation over permeable stretching sheet with slip condition. Complexity 2019, 2019, 3121896. [CrossRef]

27. Reza-E-Rabbi, S.; Ahmmed, S.F.; Arifuzzaman, S.; Sarkar, T.; Khan, M.S. Computational modelling of multiphase fluid flow behaviour over a stretching sheet in the presence of nanoparticles. Eng. Sci. Technol. Int. J. 2020, 23, 605-617. [CrossRef]

28. Sakiadis, B.C. Boundary-layer behavior on continuous solid surfaces: I. Boundary-layer equations for two-dimensional and axisymmetric flow. AIChE J. 1961, 7, 26-28. [CrossRef]

29. Tamoor, M.; Shahzeb; Zaman, Q. Linear radiation effects on forced convective slip flow along a nonlinearly stretch cylinder in an absorptive medium. Alex. Eng. J. 2018, 57, 537-545. [CrossRef]

30. Tamoor, M.; Waqas, M.; Khan, M.I.; Alsaedi, A.; Hayat, T. Magnetohydrodynamic flow of Casson fluid over a stretching cylinder. Results Phys. 2017, 7, 498-502. [CrossRef]

31. Tsou, F.K.; Sparrow, E.M.; Goldstein, R.J. Flow and heat transfer in the boundary layer on a continuous moving surface. Int. J. Heat Mass Transf. 1967, 10, 219-235. [CrossRef]

32. Turkyilmazoglu, M. Dual and triple solutions for MHD slip flow of non-Newtonian fluid over a shrinking surface. Comput. Fluids 2012, 70, 53-58. [CrossRef]

33. Turkyilmazoglu, M. Heat and mass transfer of MHD second order slip flow. Comput. Fluids 2013, 71, 426-434. [CrossRef]

34. Wang, C.Y. Flow due to a stretching boundary with partial slip-an exact solution of the Navier-Stokes equations. Chem. Eng. Sci. 2002, 57, 3745-3747. [CrossRef] 
35. Wang, M.; Fu, Y. Necking of a hyperelastic solid cylinder under axial stretching: Evaluation of the infinite-length approximation. Int. J. Eng. Sci. 2021, 159, 103432. [CrossRef]

36. Waqas, M.; Farooq, M.; Khan, M.I.; Alsaedi, A.; Hayat, T.; Yasmeen, T. Magnetohydrodynamic (MHD) mixed convection flow of micropolar liquid due to nonlinear stretched sheet with convective condition. Int. J. Heat Mass Transf. 2016, 102, 766-772. [CrossRef]

37. Yasmin, A.; Ali, K.; Ashraf, M. Study of heat and mass transfer in MHD flow of micropolar fluid over a curved stretching sheet. Sci. Rep. 2020, 10, 4581. [CrossRef] 